\title{
Pre-Peritoneal Open Mesh Repair for Inguinal Hernia
}

\author{
Dr. A. S. V. Sasank ${ }^{1 *}$, Dr. Y. V. Sai Sreeniketh Reddy ${ }^{1}$, Dr. K. Lakshmana Murthy ${ }^{2}$
}

${ }^{1}$ Post Graduate, Department of General Surgery, KIMS, Narketpally, Telangana, India

${ }^{2}$ Professor \& HOD, Department of General Surgery, KIMS, Narketpally, Telangana, India

DOI: $10.36347 /$ sasjs.2020.v06i09.002

| Received: 28.03.2020 | Accepted: 04.04.2020 | Published: 09.09.2020

*Corresponding author: Dr. A. S. V. Sasank

\section{Abstract}

Many different techniques have been used to repair inguinal hernia, each with their own advantages and disadvantages. This study was done by using the principles of stoppas and laparoscopic TEP procedures but by giving small incision under spinal anaesthesia using blunt dissection for creating the pre peritoneal space. The study was done with 90 cases in Kamineni Institute of Medical Sciences, Narketpally, Nalgonda, India. Cases which were included in the study are recurrent hernias and those above the age of 18 with primary and recurrent hernias. Cases which were excluded from the study are those with complicated hernias (strangulation, obstruction) and those cases with complete hernias. The study was done over a period of 26 months with a mean operative time of U/L hernias to be $35.23 \mathrm{~min}$ and for B/L hernias to be $43.54 \mathrm{~min}$. For $\mathrm{U} / \mathrm{L}$ recurrent hernias it was 38 min and for $\mathrm{B} / \mathrm{L}$ recurrent hernias it was 46 min. Post operative complications were found in 5 cases (hematoma-2, seroma-3), there were 3 cases with reported post operative pain and one case of recurrence. By doing this study we were able to understand that the method of combining the best of effects of laparoscopic TEP and stoppas had quite a few advantages such as small incision, less operative time, less recurrence rate, patient compliance and less cost. Other advantages that were observed was that anatomy could be directly visualised which decreases the learning curve for laparoscopic TEP. Local wound infections were minimal using this procedure.

Keywords: inguinal hernia, laparoscopic TEP, anaesthesia, strangulation.

Copyright @ 2020: This is an open-access article distributed under the terms of the Creative Commons Attribution license which permits unrestricted use, distribution, and reproduction in any medium for non-commercial use (NonCommercial, or CC-BY-NC) provided the original author and source are credited.

\section{INTRODUCTION}

Anterior repair is the most common operative approach for inguinal hernias. Tension free repairs are now standard and there are a variety of different types. Older tissue repairs are rarely indicated except for patients with contamination or concomitant bowel resection, where placement of prosthetic mesh is contraindicated.

Anterior approaches have some disadvantages in terms of long operative time, seroma formation, nerve damage, ischemic orchitis, injury to the vas deferens and recurrence, due to which the posterior approaches emerged. Conventional posterior approaches are stoppas, laparoscopic TEP and TAPP procedures.

Laparoscopic TEP and TAPP procedures have a long learning curve, costly dissecting balloons and requirement of general anaesthesia
The stoppas procedure (GPRVS) is done by wrapping the lower part of the parietal peritoneum with prosthetic mesh used for recurrent and bilateral inguinal hernias but has the disadvantage of big incision and extensive tissue dissection.

We used the principles of stoppas and laparoscopic TEP procedures but by giving small incision under spinal anaesthesia using blunt dissection for creating the pre-peritoneal space.

\section{MATERIALS AND METHODS}

This is a study of 90 cases in Kamineni institute of medical sciences, Narketpally, Nalgonda dist. India. Surgery was done under spinal anaesthesia. There were a total of 60 unilateral inguinal hernias of direct and indirect type, 26 bilateral inguinal hernias and 14 recurrent inguinal hernias.

\section{Inclusion Criteria}

- recurrent hernias

- Age more than 18 years with primary and recurrent hernias 


\section{Exclusion Criteria}

- Complicated hernias (strangulation, obstruction)

- Complete hernias.

\section{PROCEDURE}

Procedure principle is placement of prosthetic mesh occluding the myopectineal orifice of fruchaud by pre-peritoneal approach with a small incision and proper haemostasis. Procedure is done in supine position under spinal anaesthesia. A midline incision is taken just above the pubic symphysis measuring $3-4 \mathrm{~cm}$ with the upper end of incision at the level of anterior superior iliac spine (ASIS). Incision is deepened and the linea alba is cut in the line of incision, the two recti muscles are split and the preperitoneal space is created by retraction and blunt dissection as far as iliopsoas fascia and ASIS. Dissection is continued in the retropubic space of Retzius infront of the bladder. Direct sacs are reduced in the course of dissection spontaneously. The sacs of indirect hernias are identified after delivering the cord structures through the main wound. Sac is carefully separated from the cord structures avoiding injury to the testicular vessels. Small indirect sacs are reduced easily, but large indirect sacs are divided with diathermy near internal inguinal ring leaving the distal sac in situ. Any accidentally opened peritoneum is closed with 2-0 absorbable suture.
After all hernias are reduced a $12 \times 14 \mathrm{~cm}$ piece of polypropylene mesh is placed in the inguinal region and evenly spread and the mesh is made to cross the midline by a minimum of $4 \mathrm{~cm}$. The mesh is fixed to the pubic tubercle and posterior rectal musculature (optional) and the patient is asked to take a deep breath by and by this the mesh will spread evenly by Pascal's law. Mesh should cover the direct, indirect, and femoral spaces. No 14 suction drain is kept in-situ. Incision is closed in layers. Compression dressing is placed on the operated area. Post operative complications like hematoma, seroma and infections were noted. Suture removal is done on $10-12^{\text {th }}$ post operative day.

\section{RESULTS}

One hundred inguinal hernia patients were operated over a period of 26 months by this method of which are 60 unilateral, 26 bilateral and 14 recurrent inguinal hernias. The mean operative time recorded for $\mathrm{U} / \mathrm{L}$ hernias is $35.23 \mathrm{~min}$ for bilateral hernias is 43.54 min. and $38 \mathrm{~min}$ and $46 \mathrm{~min}$ for $\mathrm{U} / \mathrm{L}$ and $\mathrm{B} / \mathrm{L}$ recurrent hernias respectively. Post operative complications like hematoma formation noted in 2 patients. seroma formation noted in 3 patients, post operative pain noted in 3 patients and. recurrence noted in 1 patient (b/l recurrent hernia case.)

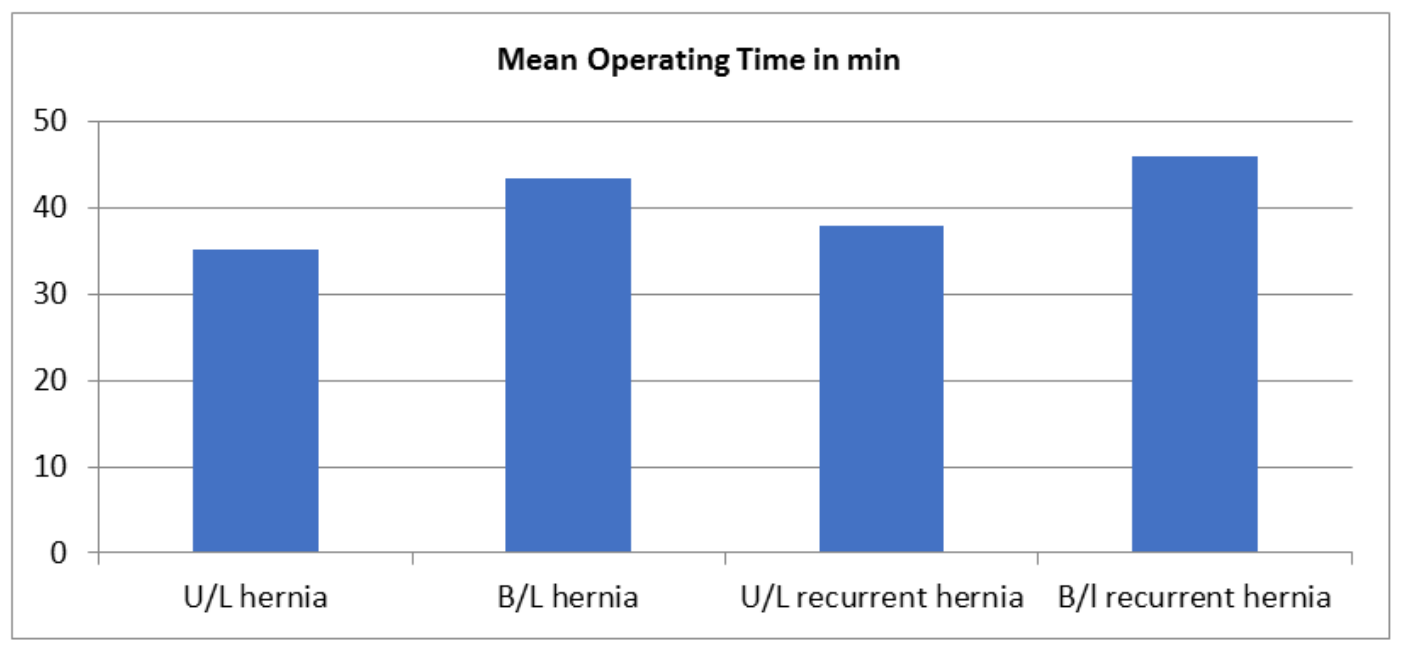

\section{DISCUSSION}

Strengthening the posterior inguinal floor is the main principle for inguinal hernia surgery. Lichenstiens tension free repair is the most standard procedure which is done all over the world with least recurrence rate $(0.3 \%)$. It is widely accepted as it has advantages like non requirement of specialized surgical equipment and very low recurrence rates. But complications like Hematoma formation, Seroma Formation, Nerve Injuries, nerve entrapments, injury to the testicular vessels and vas deferens, testicular atrophy, Scrotal oedema are more common with this anterior dissections. And for bilateral hernias we have to use two separate incisions .For recurrent cases it is difficult to go through anterior approach because of scaring of tissues.

To prevent these posterior complications posterior approaches are emerged. Stoppas (Giant prosthetic reinforcement of visceral sac), TEP (Total extra peritoneal repair) and TAPP (Trans abdominal pre-peritoneal repair) are common posterior approaches. Pre-peritoneal approaches are better in terms of avoiding local wound complications, for bilateral cases repair is done through the same incision or ports. And for recurrent cases it is advisable to go for posterior approach. 
Stoppas procedure is done in cases of recurrent and bilateral inguinal hernias requires big incision with extensive tissue dissection of pre peritoneal space for the insertion of mesh. The potential complications like fluid collections due to extensive tissue dissection are hematoma, seroma, infection. Coda et al., [1] reported a $24.6 \%$ rate of hematoma and seroma. Solorzoo et al., 1999 reported $14 \%$ hematoma infectious complications while Beeys et al., [2] reported $22.6 \%$ of hematoma and seroma, hydrocoele, hematoma, and the use of suction drainage in $83 \%$ of the patients prolonged the days of hospital stay with a mean of 3.5 days.

Laparoscopic procedures total extra peritoneal repair (TEP) is done by one umbilical camera port and two working ports. The procedure done under general anaesthesia. Pre-peritoneal space is created by balloon dissection the hospital cost of laparoscopic repairs is significantly higher than that of conventional repair because of expensive equipment needs and general anesthesia is required for laparoscopy adding complications of general anesthesia.

But in Open TEP which is done under spinal anaesthesia, with a small incision, not using specialized equipments. Direct visualization of anatomy and not disturbing the inguinal Canal structures like testicular vessels, vas differens and nerves are the main advantages of this procedure. The learning curve for laparoscopic TEP is minimised with this open procedure by understanding the anatomical relations of vital structures, open TEP is especially useful for recurrent hernia which are approached anteriorly in previous surgeries.

Post operative pain is seen 2 cases (2\%), for whom oral analgesics are given. Local wound complications like hematoma, seroma formation is seen in 4 patients (4\%), comparitively very much lesser incidence thus lichenstein repair. Recurrence was seen one case $(1 \%)$ for which open lichenstein's repair done after 6 months (based on studies by Amid PK [3] that a shrinkage reduces it's size by $20 \%$ during the first 6 months proposes the need to place mesh with larger size to prevent such recurrences. The mesh should not be smaller than $24.5 \mathrm{cms}$ which is the distance between two iliac spines and navel to pubis.

Since repair is done through midline incision it is convenient in cases for bilateral hernias to be dealt with through the same incision and there is good patient compliance in terms of pain, edema, swelling as compared to lichenstein repair.

\section{CONCLUSION}

Looking into the advantages and disadvantages the above mentioned techniques we performed the method combining the best effects of laparoscopic TEP and stoppas in terms like spinal anaesthesia, small incision, less operative time, less recurrence rate with good cosmesis and patient compliance and less cost. Here we can visualize the anatomy directly which decreases the learning curve for laparoscopic TEP, local wound infection like hematoma, seroma, infections, neurodynia, ischemic orchitis are negligible with this procedure.

\section{REFERENCES}

1. Coda BA, O'Sullivan B, Donaldson G, Bohl S, Chapman CR, Shen DD. Comparative efficacy of patient-controlled administration of morphine, hydromorphone, or sufentanil for the treatment of oral mucositis pain following bone marrow transplantation. Pain. 1997 Sep 1;72(3):333-46.

2. Beets GL, Dirksen CD, Go PM, Geisler FE, Baeten CG, Kootstra G. Open or laparoscopic preperitoneal mesh repair for recurrent inguinal hernia?. Surgical endoscopy. 1999 Apr 1;13(4):323-7.

3. Amid PK. Classification of biomaterials and their related complications in abdominal wall hernia surgery. Hernia. 1997 May 1;1(1):15-21. 